\title{
Nonlinear features identified by Volterra series for damage detection in a buckled beam
}

\author{
S. B. Shiki ${ }^{1, a}$, C. Hansen ${ }^{1, b}$, and S. da Silva ${ }^{1, c}$ \\ ${ }^{1}$ UNESP - Univ Estadual Paulista, Faculdade de Engenharia de Ilha Solteira, \\ Departamento de Engenharia Mecânica, Av. Brasil 56, 15385-000, Ilha Solteira, SP, Brasil.
}

\begin{abstract}
The present paper proposes a new index for damage detection based on nonlinear features extracted from prediction errors computed by multiple convolutions using the discrete-time Volterra series. A reference Volterra model is identified with data in the healthy condition and used for monitoring the system operating with linear or nonlinear behavior. When the system has some structural change, possibly associated with damage, the index metrics computed could give an alert to separate the linear and nonlinear contributions, besides provide a diagnostic about the structural state. To show the applicability of the method, an experimental test is performed using nonlinear vibration signals measured in a clamped buckled beam subject to different levels of force applied and with simulated damages through discontinuities inserted in the beam surface.
\end{abstract}

\section{Introduction}

Nonlinear behavior is commonly found in structures due to several sources and many effects as jumps, gaps, discontinuities, harmonics, beyond others appear frequently in the responses of structures caused by excitation condition, large displacement, new materials, etc $[8,9,2]$. Additionally, structural damages also induce nonlinear behavior, for instance cracks, delamitation, buckling and postpuckling, beyond other examples [10]. Thus, if the monitored system operates initially with linear behavior, the existence of damage is easily detected with some features that can identify nonlinear behavior. However, a common situation is when the system is initially nonlinear in the undamaged state. In this scenario, most existing structural health monitoring (SHM) methods are inadequate. Bornn et al. [1] exemplified the inadequacy of SHM linear-based methods to treat nonlinear systems. In that paper, the authors proposed autoregressive support vector machine using times series for monitoring the initially nonlinear system.

In the present work is shown a recent method based on Volterra series proposed by the authors for SHM application [4]. The procedure is able to detect the nonlinear behavior and can be used for monitoring the structural health of the system even if the level of nonlinearity in the undamaged condition is high. The novelty of the present paper is the application of this approach with experimental data extracted from a test rig involving a buckled beam.

The paper starts with a quick description of the discretetime Volterra for SHM application. Next the identified nonlinear features are used to compute a damage-sensitive index. The application in a buckled beam with damages induced by locally decreasing the stiffness through a cut in the beam surface is presented. Finally, the concluding remarks are presented.

\footnotetext{
a e-mail: sbshiki@gmail.com

b e-mail: engcristianhansen@gmail .com

c e-mail: samuel@dem.feis.unesp.br
}

\section{Discrete-time Volterra series}

The response $y(k)$ of a nonlinear system can be well approximated by discrete-time Volterra series using multiple convolutions [3]:

$$
y(k)=\sum_{\eta=1}^{+\infty} \mathscr{H}_{\eta}(k)=y_{1}(k)+y_{2}(k)+y_{3}(k)+\cdots
$$

where $y_{1}(k), y_{2}(k), y_{3}(k), \cdots$ are the linear, quadratic, cubic and so on contributions of the output $y(k)$ in $k=1, \cdots, K$ ( $K$ is the number of time samples) and $\mathscr{H}_{\eta}(k)$ is the Volterra functional given by multidimensional convolutions:

$$
\mathscr{H}_{\eta}(k)=\sum_{n_{1}=0}^{N_{1}} \ldots \sum_{n_{\eta}=0}^{N_{\eta}} \mathcal{H}_{\eta}\left(n_{1}, \ldots, n_{\eta}\right) \prod_{i=1}^{\eta} u\left(k-n_{i}\right)
$$

where $u(k)$ is the input signal and $\mathcal{H}_{\eta}\left(n_{1}, \ldots, n_{\eta}\right)$ are the $\eta$ th-order Volterra kernels considering the truncated values $N_{1}, \ldots, N_{\eta}$ for each kernel.

However, the number of samples $N_{1}, \ldots, N_{\eta}$ is high because the practical systems have large memories and the identification of the kernels $\mathcal{H}_{\eta}\left(n_{1}, \ldots, n_{\eta}\right)$ is ill-posed and with serious problems with convergence. Fortunately, the Volterra kernels can be expanded using Kautz functions to overcome these drawbacks:

$$
\mathcal{H}_{\eta}\left(n_{1}, \ldots, n_{\eta}\right) \approx \sum_{i_{1}=1}^{J_{1}} \ldots \sum_{i_{\eta}=1}^{J_{\eta}} \mathcal{B}_{\eta}\left(i_{1}, \ldots, i_{\eta}\right) \prod_{j=1}^{\eta} \psi_{i_{j}}\left(n_{j}\right)
$$

where $J_{1}, \cdots, J_{\eta}$ are the number of samples in each orthonormal projections of the Volterra kernels $\mathcal{B}_{\eta}\left(i_{1}, \ldots, i_{\eta}\right)$ and and $\psi_{i_{j}}\left(n_{i}\right)$ are the Kautz functions that are appropriate for the representation of underdamped oscillatory systems.

Details about the Kautz functions and how to use it for nonlinear mechanical systems identification can be found in [7] and [5]. It is important to see that the order of projection $\mathcal{B}_{\eta}\left(i_{1}, \ldots, i_{\eta}\right)$ is lower and easier to obtain than the order of Volterra kernel given by $\mathcal{H}_{\eta}\left(n_{1}, \ldots, n_{\eta}\right)$ [6]. 
Thus, it is possible to rewrite the eq. (1) based on the orthonormal Kautz basis:

$$
y(k) \approx \sum_{\eta=1}^{+\infty} \mathscr{B}_{\eta}(k)
$$

where $\mathscr{B}_{\eta}(k)$ is the $\eta-t h$ orthonormal Volterra functional operator:

$$
\mathscr{B}_{\eta}(k) \approx \sum_{i_{1}=1}^{J_{1}} \ldots \sum_{i_{\eta}=1}^{J_{\eta}} \mathcal{B}_{\eta}\left(i_{1}, \ldots, i_{\eta}\right) \prod_{j=1}^{\eta} l_{i_{j}}(k)
$$

that is a multiple convolution between the orthonormal kernel given by $\mathcal{B}_{\eta}\left(i_{1}, \ldots, i_{\eta}\right)$ and $l_{i_{j}}(k)$, that is a simple filtering of input signal $u(k)$ by the Kautz function $\psi_{i_{j}}\left(n_{i}\right)$ :

$$
l_{i_{j}}(k)=\sum_{n_{i}=0}^{V-1} \psi_{i_{j}}\left(n_{i}\right) u\left(k-n_{i}\right)
$$

where $V=\max \left\{J_{1}, \ldots, J_{\eta}\right\}$.

The values of orthonormal Volterra kernel $\mathcal{B}_{\eta}\left(i_{1}, \ldots, i_{\eta}\right)$ can be grouped in a vector $\boldsymbol{\Phi}$ and can be found by solving:

$$
\boldsymbol{\Phi}=\left(\boldsymbol{\Gamma}^{T} \boldsymbol{\Gamma}\right)^{-1} \boldsymbol{\Gamma}^{T} \mathbf{y}
$$

where the matrix $\boldsymbol{\Gamma}$ contains $l_{i_{j}}(k)$ and $\mathbf{y}=[y(1) \cdots y(K)]$. It is worth to note that $\eta$ can be usually truncated in 3 kernels to represent the most part of the structural nonlinearities with smooth behavior.

\section{Damage detection approach}

If a healthy state is known, the Volterra kernels can be identified and used as reference based on the output measured in this condition. So, the reference state can be estimated by:

$$
y_{\text {ref }} \approx \sum_{\eta=1}^{3} \mathscr{B}_{\eta}(k)=\underbrace{y_{1, \text { ref }}}_{\text {linear }}+\underbrace{y_{2, \text { ref }}+y_{3, \text { ref }}}_{\text {non-linear }}
$$

where 3 kernels were considered. If we compare the prediction error between the experimental output $y_{\exp }$ with the reference estimated by eq. (6), given by:

$$
e_{\text {ref }}=y_{\text {exp }}-y_{\text {ref }}
$$

it is expected that the statistical difference should be not significant. Now, if an unknown structural condition is measured, given by $x_{\text {exp }}$, the same Volterra model can be used to try to estimate it:

$$
x_{\text {unk }} \approx \sum_{\eta=1}^{3} \mathscr{B}_{\eta}(k)=x_{1, \text { unk }}+x_{2, \text { unk }}+x_{3, \text { unk }}
$$

If there is no damage in the system, the reference Volterra model can be able to estimate correctly the behavior. So, the prediction error:

$$
e_{\text {unk }}=x_{\text {exp }}-x_{\text {unk }}
$$

should be low and close to the reference error given by eq. (7). A feature index for damage detection can be extracted through [4]:

$$
\lambda=\frac{\sigma\left(e_{u n k}\right)}{\sigma\left(e_{r e f}\right)}
$$

where $\sigma(0)$ is the standard deviation operator. However, it is worth to observe that the prediction error can be described separately considering the linear and nonlinear contributions given by Volterra kernels. This property allows to monitor the linear and nonlinear terms separately. Thus, we can define three different damage-sensitive index:

$$
\begin{aligned}
\lambda_{\text {lin }} & =\frac{\sigma\left(e_{1, \text { unk }}\right)}{\sigma\left(e_{1, \text { ref }}\right)} \\
\lambda_{\text {quad }} & =\frac{\sigma\left(e_{2, \text { unk }}\right)}{\sigma\left(e_{2, \text { ref }}\right)} \\
\lambda_{\text {cub }} & =\frac{\sigma\left(e_{3, \text { unk }}\right)}{\sigma\left(e_{3, \text { ref }}\right)}
\end{aligned}
$$

where each error in reference condition is computed using:

$$
\begin{aligned}
& e_{1, \text { ref }}=y_{\text {exp }}-y_{1, \text { ref }} \\
& e_{2, \text { ref }}=y_{\text {exp }}-y_{1, \text { ref }}-y_{2, \text { ref }} \\
& e_{3, \text { ref }}=y_{\text {exp }}-y_{1, \text { ref }}-y_{2, \text { ref }}-y_{3, \text { ref }}
\end{aligned}
$$

and each error in unknown condition is calculated by:

$$
\begin{aligned}
& e_{1, \text { unk }}=x_{\text {exp }}-x_{1, \text { unk }} \\
& e_{2, \text { unk }}=x_{\text {exp }}-x_{1, \text { unk }}-x_{2, \text { unk }} \\
& e_{3, \text { unk }}=x_{\text {exp }}-x_{1, \text { unk }}-x_{2, \text { unk }}-x_{3, \text { unk }}
\end{aligned}
$$

The next section presents an experimental application to show this approach.

\section{Application in a buckled beam}

Figure (1) shows an experimental setup used to illustrate the method. It is a test rig formed by a clamped beam with dimensions of $460 \times 18 \times 2 \mathrm{~mm}$ and with a load applied on the top. The nonlinearity is actioned by geometrical effect caused by the buckling load applied. A controlled electrodynamic shaker is used to excite the beam considering different levels of amplitude measured by a force sensor.

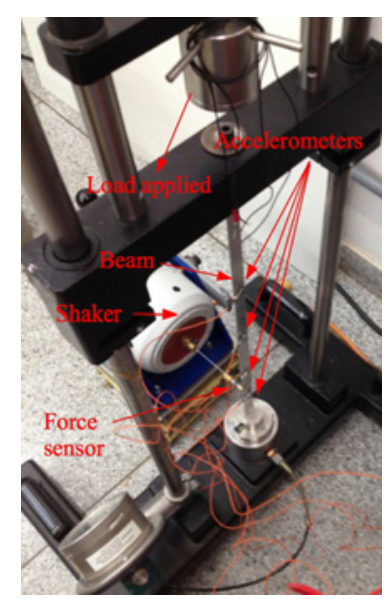

Fig. 1. Experimental setup of the clamped buckled beam. 
The velocity in the center of the beam is measured by using a laser sensor. Four accelerometers are also used to measure the vibration along the beam, but are not used in the analysis of this work. Firstly some tests are made in the undamaged condition in order to show the nonlinear behavior. A sinusoidal signal is applied in the shaker sweeping up from 20 to $50 \mathrm{~Hz}$ in order to excite the first natural frequency. Three different levels of amplitude in the signal generator, named by low $(0.01 \mathrm{~V})$, medium $(0.05 \mathrm{~V})$ and high $(0.1 \mathrm{~V})$ are used. All data acquisition is performed considering a sampling rate of $1024 \mathrm{~Hz}$ and 4096 samples.

Figure (2) presents the FRF (frequency response function) varying the amplitude applied in the shaker. Clearly it is observed a change of the resonance frequency when the input force is increased that is caused by a nonlinear effect.

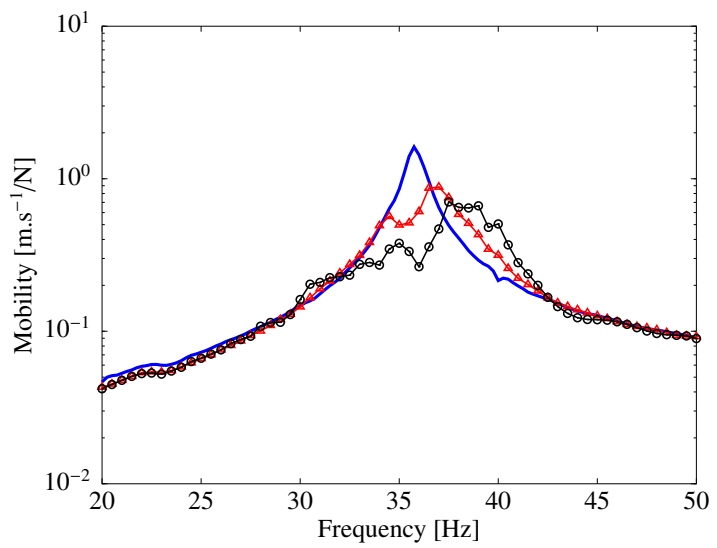

Fig. 2. FRF considering a sinusoidal sweeping up with different amplitude levels. The continuous line is the low input $(0.01 \mathrm{~V}), \Delta$ is the medium input $(0.05 \mathrm{~V})$ and $\circ$ is the high input $(0.10 \mathrm{~V})$.

Similar hardening nonlinear effect and jump can be also seen in fig. (3) when it is performed a stepped sine test using a frequency resolution of $0.1 \mathrm{~Hz}$.

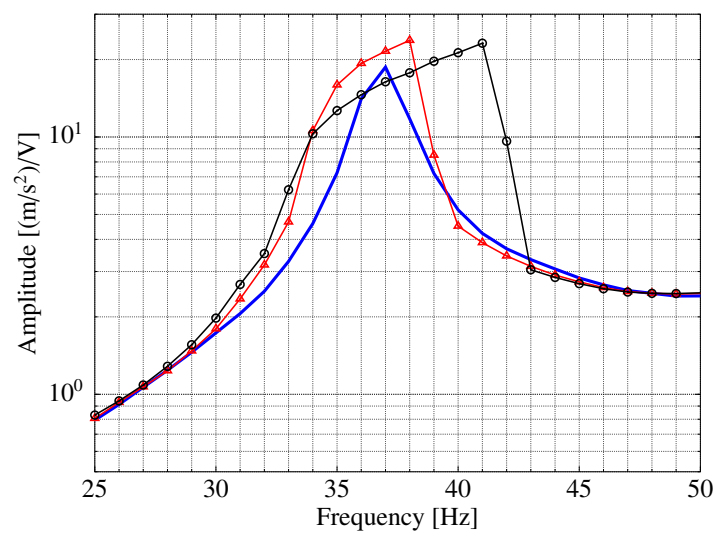

Fig. 3. Stepped sine showing the jump effect. The continuous line is the low input $(0.01 \mathrm{~V}), \Delta$ is the medium input $(0.14 \mathrm{~V})$ and $\circ$ is the high input $(0.20 \mathrm{~V})$

The three orthonormal Volterra kernels are extracted to have the reference model using the healthy dataset. The identification is performed in two steps, firstly the $\mathcal{B}_{1}\left(i_{1}\right)$ is estimated through the low input (linear part) and after $\mathcal{B}_{2}\left(i_{1}, i_{2}\right)$ and $\mathcal{B}_{3}\left(i_{1}, i_{2}, i_{3}\right)$ are estimated using the high input (nonlinear). Figure (4) shows the validation using the power spectral density (PSD) of the reference Volterra model using a sinusoidal input with frequency of $35 \mathrm{~Hz}$.

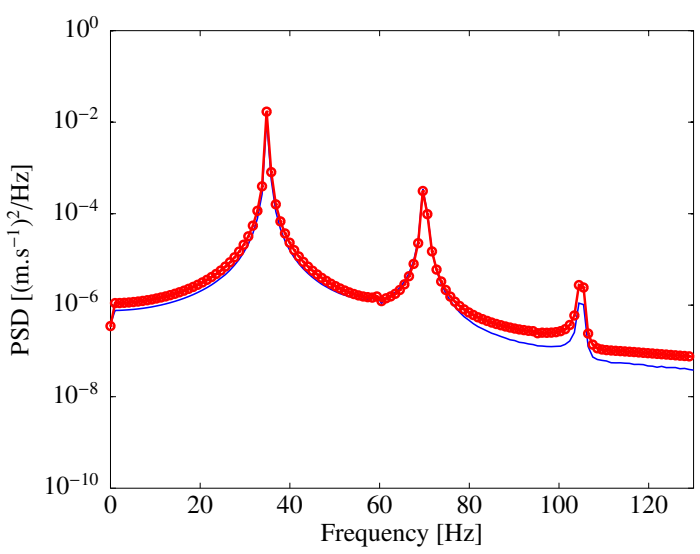

Fig. 4. PSD of the measured healthy response in comparison to the one estimated by the reference Volterra model. The continuous line is the experimental data and $\circ$ is the response estimated by the Volterra model.

The damage is induced by a cut in the beam surface 50 $\mathrm{mm}$ from the stinger connection as seen in fig. (5). Three different cuts are inserted with depth growing up, named by Damage I $(0.5 \mathrm{~mm})$, Damage II $(0.75 \mathrm{~mm})$ and Damage III $(1.0 \mathrm{~mm})$. The reference condition (healthy) is named by 0 . In each structural condition, the same excitation signal is applied in the shaker with three different amplitude levels totalizing twelve datasets for the analysis.

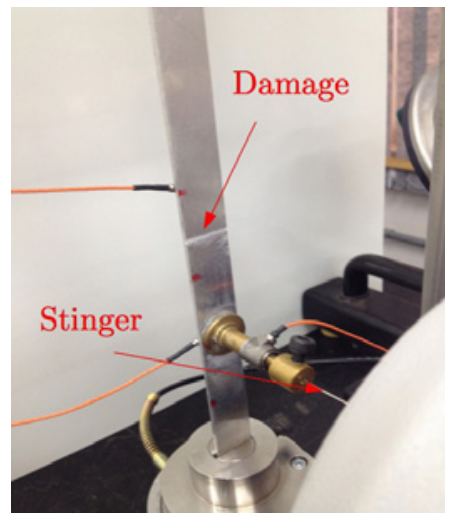

Fig. 5. Damage inserted by a cut in the beam.

The prediction errors can be analyzed in order to detect modifications in the structural behavior of the beam. Instead of observing the time-domain prediction errors, the set of $\lambda$ indexes can be used to detect damages. These indexes are based on a ratio of the standard deviation of the error in the unknown condition with the error in the reference state. So, a significant deviation from 1 can point out the presence of damage in the structure. Figure (6) shows the evolution of the $\lambda_{\text {lin }}, \lambda_{\text {quad }}$ and $\lambda_{\text {cub }}$ indexes comparing the reference and the three damaged states.

In all the input amplitudes tested is possible to see the increase in the deviation of the indexes with the increase of the depth of the cut inserted in the beam. The indexes $\lambda_{\text {lin }}$ and $\lambda_{\text {quad }}$ are also equal because the quadratic term of the response of the Volterra model identified is not significant (absence of asymmetric effects). Another important 
observation is that the $\lambda_{\text {cub }}$ is considerably more sensitive to the structural change with the increase of the input amplitude. Besides that, the linear index $\lambda_{\operatorname{lin}}$ is not sensitive to the damage when the amplitude force is high because the nonlinearity dominates the system in this condition.

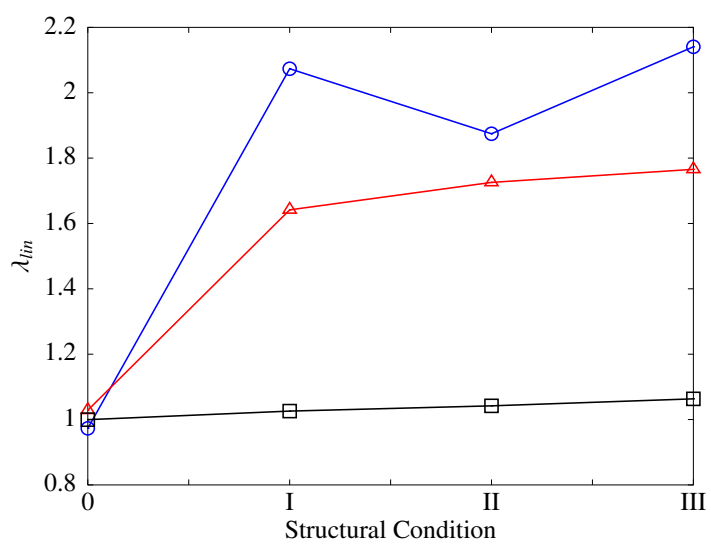

(a) Linear index $\left(\lambda_{\text {lin }}\right)$

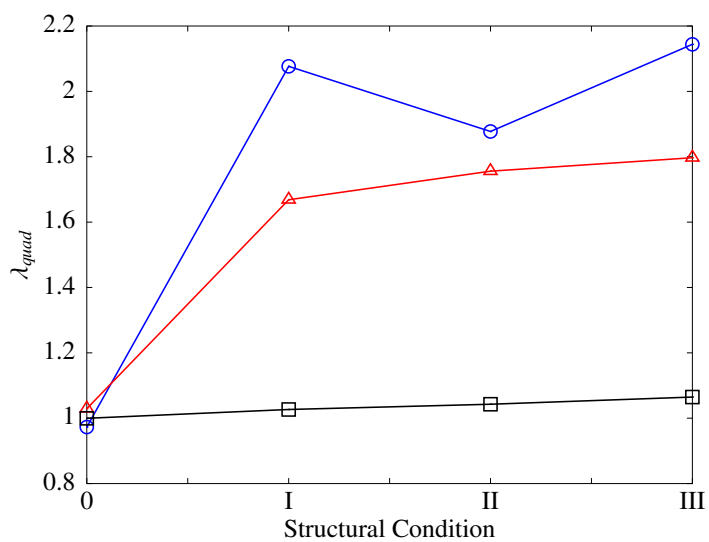

(b) Quadratic index $\left(\lambda_{\text {quad }}\right)$

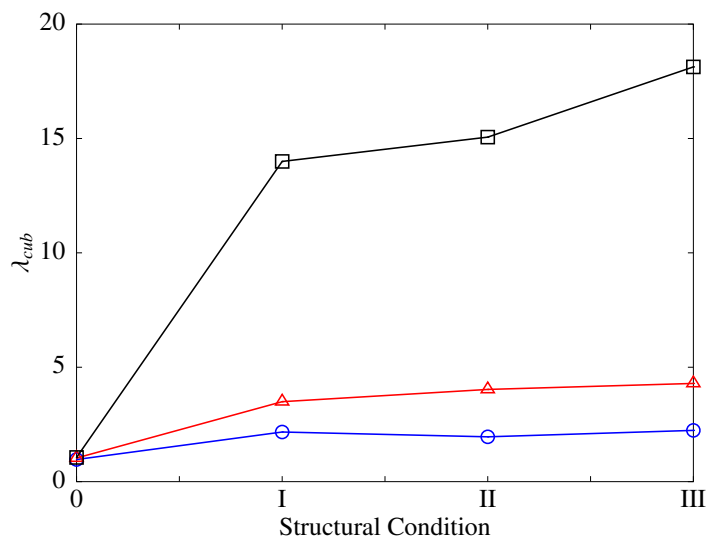

(c) Cubic index $\left(\lambda_{\text {cub }}\right)$

Fig. 6. Linear and nonlinear damage-sensitive index in three different input levels. $\bigcirc$ is the low input $(0.01 \mathrm{~V}), \triangle$ is the medium input $(0.05 \mathrm{~V})$ and $\square$ is the high input $(0.1 \mathrm{~V})$.

The cuts applied in the structure represent mostly a variation in the linear parameters of the structure caused by loss of mass and stiffness, but the nonlinear stiffness is also affected. For this reason, the coefficient $\lambda_{c u b}$ composed by the $y_{1}, y_{2}$ and $y_{3}$ is the best because it considers both the linear and nonlinear effects. Consequently, the $\lambda_{c u b}$ proposed is more sensitive to small changes in the beam.

\section{Final remarks}

The results have found that the Volterra series can separate the linear and nonlinear contributions of the vibration response and also provide a full diagnostic about the structural conditions. The nonlinear damage indexes showed to be more efficient to the structural changes than the linear one, specially when the beam tested operated in nonlinear state. The statistical reliability of the nonlinear damagesensitive index using Volterra series should be investigated in further works.

\section{Acknowledgements}

The authors acknowledge the financial support provided by Research Foundation of São Paulo (FAPESP, Brasil) by the grant number 12/09135-3 and the National Council for Scientific and Technological Development (CNPq, Brasil) by the grant number 470582/2012-0. The first and the second author are thankful to FAPESP for their scholarships grant number 12/04757-6 and 13/09008-4, respectively. The authors also thank the CNPq and FAPEMIG for partially funding the present research work through the National Institute of Science and Technology in Smart Structures in Engineering (INCT-EIE).

\section{References}

1. L. Bornn, C. R. Farrar, G. Park, International Journal of Engineering Science 48, (2010) 909-920

2. G. Kerschen, K. Worden, A. F. Vakakis, and J. C. Golinval, Mechanical Systems and Signal Processing 20, (2006) 502-592.

3. M. Schetzen, The Volterra and Wiener theories of nonlinear systems (New York: Wiley, 1980) 618 pages

4. S. B. Shiki, V. Lopes Jr and S. da Silva, Key Engineering Materials 569-570, (2013) 876-883

5. S. da Silva, S. Cogan and E. Foltête, Mechanical Systems and Signal Processing 24, (2010) 52-58

6. S. da Silva, International Journal of Non-Linear Mechanics 46, (2011) 312-320

7. S. da Silva, Mechanical Systems and Signal Processing 25, (2011) 1103-1111

8. L.N. Virgin, Introduction to Experimental Nonlinear Dynamics (Cambridge University Press, 2000), 272 pages

9. K. Worden and G. R. Tomlinson. Nonlinearity in Structural Dynamics (London : Institute of Physics Publishing, 2001) 659 pages

10. K. Worden, C. R. Farrar, J. Haywood and M. Todd, Structural Control and Health Monitoring 15, (2008) 540-567 